\title{
Migrating Opiate Pump: Atypical Cause of Meralgia Paresthetica
}

\author{
Robert E. Windsor, MD, and Samuel Thampi, MD
}

This is a case report of a 60 year-old female who presented with pain on the anterolateral aspect of her right thigh. The patient had a history of placement of a Drug Administration System (DAS, Opiate pump) in August, 1998 for chronic lumbar radiculopathy and a multiply operated on spine which she had suffered with since October, 1991. She presented with the subacute onset of focal pain on the anterior aspect of her right superior iliac crest region and dysesthesias on the anterolateral aspect of her right thigh. There was no his-

The treatment of chronic lumbar radiculopathy (failed back surgery syndrome) (FBSS) has changed significantly with the advent of implantable Drug Administration System (DAS, Opiate Pump). Studies have shown that DAS is successful in reducing pain and improving the quality of life in patients with FBSS $(1,2)$.

A DAS is typically placed in the right lower quadrant of the abdomen within the subcutaneous fatty tissue of the abdominal wall. It is typically suspended by suturing the pump to the dense subcutaneous fascia or fascia of the rectus abdominus. An opiate pump in this location is relatively free from compressing vulnerable structures such as vessels or nerves. The present case describes a patient who developed lateral femoral cutaneous nerve compression from the distal migration of a Medtronic DAS. She underwent surgery for revision of the pouch site and this ameliorated her symptoms.

\section{Case Report}

A 60-year-old white female suffered a work related back injury in 1983. She un-

From Georgia Pain Physicians and Emory University Atlanta, Georgia. Address Correspondence: Robert E. Windsor, MD, Georgia Pain Physicians

2550 Windy Hill Rd., Suite 215, Marietta, Georgia30067. E-mail: rwindsor@aol.com

Funding: There was no external funding in preparation of this manuscript. tory of recent trauma, worsening low back pain, or any other sensori-motor change. There was no history of anterior iliac crest bone graft for her spinal fusion and she had no anterolateral thigh symptoms related to her lumbar radiculopathy. Her neurological examination was significant only for decreased sensation on the anterolateral aspect of her right thigh. On abdominal examination the DAS pump was found at a low-lying location and was closely abutting the anterior and medial aspect of her superior iliac spine. She underwent revision of the

derwent lumbar laminectomy at L3-S1, anterior interbody fusion at L3-S1, with autologous right posterior iliac crest graft in October 1991 without improvement of her symptoms. She underwent a myriad of non-interventional and interventional pain treatments over the next several years without any prolonged benefit. She presented to our clinic first in 1998 at which time a detailed history, physical and psychological examination was performed. It was determined that she was not a surgical candidate; that she was experiencing a great deal of pain on a daily basis which was interfering with her function and quality of life; that she was intolerant of oral medications at a dose that would be effective at controlling her pain; and that, other than situational depression, she was not suffering from significant psychopathology. As a result of the above, it was decided to infuse Intrathecal morphine to see if she would be amenable to this form of therapy. The intrathecal morphine trial was very effective at relieving her pain and thus a DAS was implanted. Over the last 5 years, the DAS has been effective at controlling her pain such that she has not required significant oral narcotic medication or other pain treatments.

In March, 2002 she presented to our clinic with pain and paresthesias of 2-3 weeks duration pain and numbness on
DAS pump site with obliteration of the inferior and lateral aspect of the pump pocket. Her symptoms improved by $30 \%$ immediately and completely dissipated within two month following the procedure. This is the first reported case of lateral femoral cutaneous nerve neuropathy with DAS use. Recognition of focal compressive neuropathy by distal migration of a DAS is important, as they are potentially treatable with recognition.

Keywords: Meralgia paresthetica, opiate pump, Drug Administration System

the anterolateral aspect of her right thigh. She denied any history of trauma. She had presented to her primary care physician 1-2 weeks prior to coming to our clinic who injected her trochanteric bursa with a presumed diagnosis of trochanteric bursitis. The bursal injection did not relieve her symptoms. She presented for routine follow-up visit for her DAS pump programming and refill at which time the thigh pain complaints were made.

Her medical history was significant for stable coronary artery disease, irritable bowel syndrome, and peptic ulcer disease. She had no history of diabetes. In general, she was a friendly, alert, elderly thin female with attenuated soft tissues. Neurologic examination revealed that Cranial Nerves III - XII were intact. Sensorimotor exam unrelated to presenting complaints was grossly intact. No cerebellar or long tract finding were identified. She was found to have decreased fine touch sensation on the anterolateral aspect of her right thigh which did not cross the midline of the anterior or posterior thigh and did not cross the knee. There was a positive Tinel's sign at the anterior superior iliac spine and there was significant pain to gentle palpation of this region. Hip examination was unremarkable. Lumbar spine examination was essentially unchanged since her prior visit and straight leg rais- 
ing test was negative. With a clinical impression of meralgia paresthetica, the anterior superior iliac spine was surveyed and the DAS was found lying close to the anterior superior iliac spine which was accentuated in the standing position. In the supine position, abdominal examination showed the DAS at a more distal location in reference to the scar of DAS pouch in the right lower quadrant of the abdominal wall than was typical. Electrodiagnostic examination was contemplated but not performed on the patient's request however local diagnostic injection was performed which provided excellent shortterm pain relief which confirmed the diagnosis. As a result, it was planned that the pocket would be appropriately revised upon preemptive replacement of her DAS pump.

The patient underwent surgery for revision of the opiate pump site and replacement of the pump. The DAS pump was explanted. The inferior and lateral aspect of the pump pocket was exposed and the dis- tal 4-5 cm's of the pocket was approximated with non-absorbable sutures which resulted in obliteration of the inferior and lateral aspect of the pocket. A Synchromed pediatric size DAS pump was connected to the pre-existing intrathecal catheter and implanted in the revised pocket. A pediatric DAS was used as it has a smaller profile and mass compared to its adult size counterpart. The DAS was suspended by suturing the DAS pouch to the rectus abdominus fascia. Post-operative course was uneventful. Routine post-op precautions with an abdominal binder to minimize seroma formation was used. She was seen during post-operative follow-up at one week, one month, and two months. At one week follow up her symptoms had abated by $30 \%$, at one month her symptoms had abated by $80 \%$ and at two months her symptoms had completely resolved.

\section{DisCUsSION}

Meralgia paresthetica is a syndrome of pain and paresthesias on the anterolat- eral aspect of the thigh due to focal compression and or entrapment neuropathy of the lateral femoral cutaneous nerve. Pioneers of this syndrome include Bernhardt (4), who first described the condition in 1895, and Roth (5) who coined the term meralgia paresthetica.

The lateral femoral cutaneous nerve is a pure sensory nerve formed by the fusion of the posterior divisions of the second and third lumbar anterior rami. The nerve passes through the psoas major muscle and emerges superior to the iliac crest in the posterior flank. It runs inferolaterally on the iliacus muscle and enters the thigh through a tunnel formed by the lateral attachment of the inguinal ligament and the anterior superior iliac spine. The nerve runs anterior to the sartorius muscle and divides into an anterior and posterior branch. The anterior branch becomes superficial about 10 $\mathrm{cm}$. below the inguinal ligament, and divides into branches which are distributed to the skin of the anterior and later-

Table 1. Causes of Meralgia Paresthetica

1. Acute Traumatic

a. Compression by diagonal seat belts due to sudden deceleration forces in motor vehicle accidents.

b. Pelvic fractures involving anterior superior iliac spine.

c. latrogenic - Lateral femoral cutaneous nerve injury has been reported in the following surgical procedures:

i. Anterior Iliac crest bone harvesting (9)

ii. Pelvic osteotomy

iii. Femoral artery catheterization (8)

iv. Inguinal lymph node dissection

v. Appendectomy

2. Intraabdominal mass causing local compression of the nerve:
a. Tumor invasion (4)
b. Hemorrhage
c. Abscess
d. Endometriosis: Pain recurs and abates with menses.
e. Fetal compression during the second and third trimesters

3. Extrinsic mechanical compression/ stretching of the Nerve at the ilioinguinal ligament.
a. Tight-fitting garments
b. Braces, trusses
c. Tight Trouser belt
d. Obesity
e. Ascites

4. Other causes:
a. Mono neuropathy eg. Diabetes and Herpes Zoster
b. Idiopathic causes 
al parts of the thigh, as far as the knee. The terminal filaments of this nerve frequently communicate with the anterior cutaneous branches of the femoral nerve and with the infrapatellar branch of the saphenous nerve, forming with them the patellar plexus. The posterior branch pierces the tendon of the tensor fascia lata, and subdivides into filaments which pass posterior across the lateral and posterior surfaces of the thigh, supplying the skin from the level of the greater trochanter to the middle of the thigh both posteriorly and anteriorly (6). The nerve can be compressed at several potential sites (Table 1).

Specific electrodiagnostic studies include determination of the Sensory Nerve Action Potential (SNAP) and nerve conduction velocities (NCV's) along the segment of the nerve. These values are compared to the uninvolved extremity. Electrodiagnostic study has limited diagnostic and prognostic value. (7) Injury to the lateral femoral cutnaeous nerve has been reported during cardiac catheterization. (8) Autologus bone graft harvesting from the anterior superior iliac crest has also been reported to cause lateral femoral cutaneous nerve injury. (9) However, as mentioned, the patient described in this case report did not have bone harvesting or any other surgical procedure at or near the iliac crest. An implantable DAS as a cause of lateral femoral cutaneous nerve compression has not been reported in the literature. In this case, the mechanism of compression of the lateral femoral cutaneous nerve appears to be similar to that seen in obese individuals, individuals who wear tight belts or garments, or individual who wear tool belts. The similarity in all of the conditions is that the lateral femoral cutaneous nerve may be compressed and injured medial to the anterior superior iliac spine the cutaneous nerve passes under the inguinal liga- ment (10-12).

Complications of DAS implantation published in the literature include infections, headache, hemorrhage and local seroma at the site of DAS placement. Complication of DAS dissection through the adipose tissue, though not reported in the literature, has been seen by the authors. The DAS can dissect through the subcutaneous tissue possibly due to its inherent weight, atrophy of subcutaneous connective tissue and inadequate anchoring of the DAS pump. Patients who report a "moving pump", may on clinical examination have the DAS pump either turned on its axis in a clock-wise or counter-clock wise manner, the pump may flip forward or backwards such that its posterior surface is facing anteriorly, or the pump may be slide inferiorly and be visible in a more distal location. In instances of a rotating pump in which the pump flips forward to backwards, the DAS pump may have to be manually rotated under fluoroscopic visualization in order to refill the pump. If this is impossible the DAS may have to be surgically repositioned. By rolling on its axis the pump may potentially kink the catheter and interfere with adequate drug delivery.

A DAS that moves freely through the subcutaneous tissue may compress any vulnerable structure. In this case the DAS moved caudally and compressed the lateral femoral cutaneous nerve which is an atypical cause for Meralgia Paresthetica.

\section{Conclusion}

This case illustrates an atypical cause of meralgia paresthetica caused by a lowlying opiate pump secondary to migration from its original location. Surgical revision of the abdominal pocket resulted in marked improvement of her symptoms. It is important to recognize this condition, as it is potentially treatable when recognized early.

\author{
Author Affiliation \\ Robert E. Windsor, MD \\ Program Director \& President \\ Georgia Pain Physicians \\ Emory University \\ 2550 Windy Hill Road, Suite 215 \\ Atlanta, GA 30067 \\ E-mail: rwindsor@aol.com \\ Samuel Thampi, MD \\ Georgia Pain Physicians \\ Emory University \\ 2550 Windy Hill Road, Suite 215 \\ Atlanta, GA 30067
}

\section{References}

1. Roberts LJ, Finch PM, Goucke CR et al. Outcome of intrathecal opioids in chronic noncancer pain. Eur J Pain 2001; 5:353-361.

2. Unal T, Doleys D. Intrathecal infusion systems for treatment of chronic low back and leg pain of non-cancer origin. South Med Journal 1996; 89:295-300.

3. Lagueny A, Deliac MM, Deliac P et al. Diagnostic and prognostic value of electrophysiologic tests in meralgia paresthetica. Muscle Nerve 1991; 14:51-56.

4. Bernhardt M. Uber isolirt im gebiete des N. cutaneous femoris externus vorkommende paresthesian. Neurol Centrabl $1895 ; 14: 242-244$.

5. Roth V. Meralgia paresthetica. Med Obozr 1895; 43:678.

6. William PL, Warwick R. Neurology. In William PL, Warwick R (eds). Grays' Anatomy. WB Saunders, Philadelphia, 1980, pp 1107-1108.

7. Lagueny A, Deliac MM, Deliac P et al. Diagnostic and prognostic value of electrophysiologic tests in Meralgia Paresthetica. Muscle Nerve 1991; 14:51-56.

8. Butler R, Webster MW. Meralgia paresthetica: An unusual complication of cardiac catheterization via the femoral artery. Catheter Cardiovasc Interv 2002; 56:69-71

9. Mirovsky Y, Neuwirth M. Injuries to the lateral femoral cutaneous nerve during spine surgery. Spine 2000; 25:1266-1269.

10. Ghent W. Meralgia paresthetica. Can Med Assoc / 1959; 81:631-633.

11. Stookey B. Meralgia paresthetica: Etiology and treatment. JAMA 1928; 90:1705-1707.

12. Teng P. Meralgia paresthetica. Bull LoS Angel Neurol Soc 1972; 37:75-83. 